\title{
ARAIM for Vertical Guidance Using GPS and BeiDou
}

\author{
Ahmed El-Mowafy \\ Department of Spatial Sciences, Curtin University, Australia
}

\begin{abstract}
An advanced Receiver Autonomous Integrity Monitoring (ARAIM) approach is investigated when augmenting GPS satellites with the current regional BeiDou constellation. A procedure for integrity monitoring, including checking its availability, fault detection and exclusion, and integrity testing is presented. Fault modes and their probabilities using GPS and GPS+BeiDou are discussed. Testing of ARAIM for vertical guidance using real data in eight sites distributed globally (Australia, China, Netherlands, eastern Canada and Peru) show that the addition of the BeiDou constellation, despite the decreased preliminary confidence placed in its performance compared with GPS, results in a substantial improvement to ARAIM availability performance and a higher level of integrity, in particular at sites observing all of its current constellation (Australia and China). The improvement was less in sites that can only observe some or no GEO and IGSO satellites (Netherlands, Canada and Peru). However, the benefit of adding BeiDou to GPS at these sites is expected to substantially improve with full deployment of MEO satellites.
\end{abstract}

Keywords: Integrity Monitoring, RAIM, GPS, BeiDou.

\section{Introduction}

The modernisation of GPS accompanied with the emergence of new constellations of GNSS creates an exciting prospect for using GNSS as a supplementary and ultimately a primary means of aircraft navigation. For this to happen, integrity must be assured to keep safety paramount. Integrity can be defined as the ability of the system to provide timely warnings to users as to when the system should not be used for navigation (US FRP, 2008). The space component of the GPS system provides the integrity parameter User Range Accuracy (URA) in the navigation message. URA is a main component in the range error model, which is used to produce the weighting matrix for the position solution and calculation of the vertical protection level. Galileo on the other hand broadcasts integrity information as
Signal In Space Accuracy (SISA), its Monitoring Accuracy (SISMA) and Integrity Flag (IF) (HofmannWellenhof et al., 2008). BeiDou transmits User Range Error (URE) in its navigation message (China Satellite Navigation Office, 2013). Satellite Based Augmentation Systems (SBAS) such as WAAS, EGNOS, MSAS, GAGAN and SDCM use a network of ground reference stations to detect small variations in GNSS signals and to check if the observations at a certain time meet integrity requirements. Such information is sent as a message to the aircraft via geosynchronous satellites. However, these systems are only regional. Having a global SBAS system is not feasible in the near future due to the need for a large number of geosynchronous satellites and the extra cost of establishment a global network of reference stations with SBAS standards.

Receiver Autonomous Integrity Monitoring (RAIM) is an approach that allows errors to be detected by the receiver itself by performing a consistency check of all observations and check integrity of the system (Brown, 1992; Lee et al., 2005, Hwang and Brown, 2008). RAIM was originally designed for en-route and non-precision phases of flight. To verify positioning integrity in aviation, the Navigation Sensor Error (NSE) performance must meet navigation requirements in both the lateral and vertical dimensions. However, requirements on vertical navigation sensor error (NSEV) are more demanding.

By using measurements from multiple frequencies, users would be able to eliminate the ionosphere delay error, which is the largest error source for single frequency receivers, and thus, greatly increase positioning accuracy and integrity. With integration of new GNSS constellations, the increased number of satellites in view will improve the user geometry, and the new civilian enhanced signals in L5/E5a will allow receivers to cancel the first order ionospheric delay. This has led to consider the use of RAIM for vertical guidance. The new algorithms and assumptions that could provide vertical guidance have been labelled Advanced RAIM (ARIAM) to distinguish them from traditional RAIM. 
Most RAIM/ARAIM studies have considered the use of ionosphere-free linear combination of two frequencies and the case of triple frequencies has been discussed in Guo et al. (2011). The case of RAIM using multipleconstellations has been considered in Ene et al., 2007, Lee and McLaughlin, 2007, and Lee 2012, for integrating GPS with Galileo, Choi et al. (2011a-b, 2012) using GPS and GLONASS, and some introductory work for integrating GPS with BeiDou has been presented in Lijun et al. (2012) and El-Mowafy and Arora (2013).

In this study, we will focus on ARAIM for vertical guidance and restrict attention only to a combined GPS+BeiDou system of measurements to explore RAIM performance in case of using receivers with merely such capabilities. The performance of combining the two systems will be compared with using only GPS, as GPS has, by far, the most extensive history of satellite operation. Validate of the presented ARAIM will be performed using real data. In Australia, integration of BeiDou with GPS for ARAIM is motivated by:

- BeiDou has currently 14 operational satellites and is planned to be completed within this decade.

- Australia has a location that allows for tracking most BeiDou GEO and IGSO satellites.

- Currently, there is no SBAS measurement correction or integrity service available over Australia and GPS is, in general, not good enough to support vertical guidance by itself.

- BeiDou has taken integrity as a critical design objective (Geng et al., 2010).

Although GLONASS has a current constellation of 24 satellites, it is not included in the scope of this paper and its implementation with GPS in ARAIM is still under investigation. For example, Choi et al. (2011b) showed worse RAIM accuracy for the dual constellation GPS+GLONASS compared with GPS alone as GLONASS satellites do not have the same performance characteristics as GPS satellites. However, their results in Choi et al., (2012) and Walter et al. (2013) showed some improvement when using a refined error model. The use of GLONASS will attract more attention in the future, in particular, when three open CDMA signals are broadcast by the new generation K-series satellites. Therefore, we plan in a future research to investigate the use of all systems in ARAIM, addressing the limitation of each and their possible error models.

The contribution of this paper is threefold; it presents a proposed error model and fault probabilities when combining GPS and BeiDou; it validates the ARAIM using the two constellations using real data distributed globally at selected stations, and it clearly summarizes an ARAIM process. The paper is organised as follows, a summary of an algorithm for integrity monitoring for vertical guidance is first presented. The use of GPS and BeiDou observations for ARAIM is discussed. Testing of the method and its results are next presented.

\section{Integrity Monitoring}

ARAIM is modelled to detect if the vertical positioning error goes beyond a certain threshold. If there is a faulty satellite within a constellation, then navigation using GNSS will be considered unavailable for safety reasons unless the faulty satellite can be detected and excluded from the navigation solution. This technique is known as Fault Detection and Exclusion (FDE). Using the solution separation method, a minimum of five satellites are needed to perform the navigation solution with an ability to detect a satellite anomaly, and in order to exclude a faulty satellite, a minimum of six satellites must be observed. To determine availability of ARAIM, testing should be performed to check whether a critical vertical protection level $(V P L)$ is less than the vertical alert limit (VAL) (GEAS, 2008). VPL is estimated, epoch-by-epoch, based on satellite geometry and assumed error model and parameters along with the broadcast $U R A$ values. VAL is taken $35 \mathrm{~m}$ for CATI in the precision approach. The Hazardously Misleading Information (HMI) exists when the vertical position error $(V P E)>V A L$ and $V A L>V P L$ for longer than the time-to-alert (TTA).

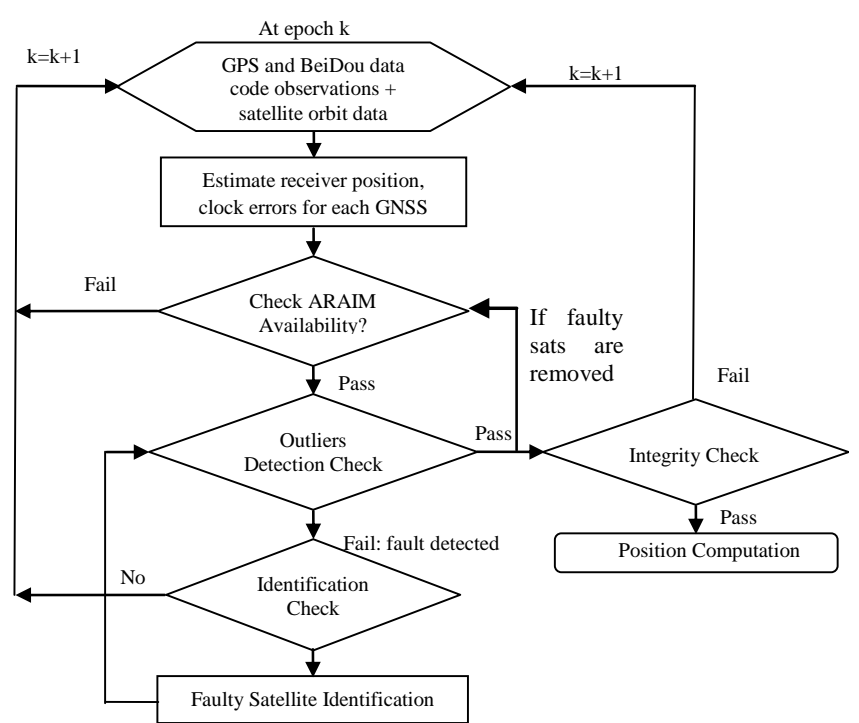

Figure 1: Flowchart of the integrated integrity monitoring algorithm

A summary of the approach followed in this study for integrity monitoring is illustrated in Figure 1. At epoch $k$, the unknown position and clock biases are first determined, followed by checking availability of ARAIM according to geometry of available satellites, assumed quality of the observations and integrity assumptions. Next, FDE approach is performed to detect and isolate faulty satellites if present in the data. 
Availability needs to be checked again if any satellite is removed when identification with faulty observations. This satellite has to be continuously checked and remained isolated from the solution for a certain period (time to recovery period) after its observations regain their quality to ensure that the cause of the fault is not recurring. Finally, integrity is checked to assess if the position error is within the allowable protection level. Another approach would be to do the FDE first followed by availability testing and finally integrity checking. The following sections describe the three steps, inspection of ARAIM availability, FDE, and integrity checking.

\subsection{Observation model and solution}

The linearised GNSS measurement model can be expressed as:

$$
y=G x+\varepsilon
$$

where $y$ is the measurement vector, taken as the difference between the observed and calculated pseudorange from the approximate values of the coordinates. $x$ refers to the difference between the final and approximate values of the unknown parameters, which include the three dimensional position and the receiver clock error for each constellation. $\varepsilon$ is the vector of random errors, which are assumed Gaussian with zero mean. The least square solution of the unknown parameters reads (Blanch, 2007):

$$
\hat{x}=\left(G^{T} W G\right)^{-1} G^{T} W y=S \times y
$$

where $W$ is the weight matrix of measurement vector $y$ computed from $U R A$, which is disseminated through the navigation message and accounts for clock and ephemeris errors, in addition to troposphere delay, multipath and receiver noise, which are modelled for instance as given in GEAS, 2010. First order ionosphere delay is eliminated by using ionosphere-free linear combination of code measurements (e.g. L1 and L5 for GPS, B1 and B2 for BeiDou). For GPS, the broadcast orbital errors is typically within $1 \mathrm{~m}$ (El-Mowafy, 2011), whereas for BeiDou satellites, the broadcast ephemerides achieve a typical user range error at the $1.5 \mathrm{~m}$ level (Montenbruck et al. 2013). Thus, BeiDou medium Earth orbit (MEO) and inclined Geosynchronous Orbit (IGSO) satellite observations are assumed to have slightly lower accuracy than GPS as shown in El-Mowafy (2013). Observations of BeiDou geostationary (GEO) satellites have significant multipath and a degraded performance in the static mode as a result of observation geometry. However, multipath tends to randomized in the airborne mode.

The direction cosine (design) matrix $G$ provides the transformation from the observation domain to the position domain. For the $i^{\text {th }}$ satellite, the corresponding row $G_{i}$ of $G$ reads (Knei $\beta 1$ et al. 2009, El-Mowafy 2005):

$$
G_{i}=\left[\begin{array}{llll}
-\cos \theta_{i} \sin \alpha_{i} & -\cos \theta_{i} \cos \alpha_{i} & -\sin \theta_{i} & 1
\end{array}\right]
$$

where $\theta_{i}$ and $\alpha_{i}$ denote the elevation angle and azimuth, determined from the broadcast satellite ephemeris or almanac and the approximate receiver location.

\subsection{ARAIM availability testing and integrity checking}

Availability is the fraction of time when integrity service is supported, that is when the protection levels are below their alert limits and the expected accuracy and continuity are within their requirements. For the system to be useful, it must be available at least $99 \%$ of the time at any location where GNSS navigation service is authorized. For scheduled service, the system may need to be available for even greater percentages of time. ARAIM with vertical guidance is declared available if $V P L<V A L$. VPL is dependent on the integrity budget, the continuity budget, the nominal error model, the a-priori probability of failure for each satellite (or set of satellites), and satellite geometry. $V P L$ is taken as the $\max \left\{V P L_{0}, \max (V P L n)\right\}$, where $V P L_{0}$ is the $V P L$ for the full set of observed satellites of a total number $N$, given as (GEAS 2010):

$$
\begin{aligned}
V P L_{0} & =\text { Gaussian term }+ \text { Bias overbound } \\
& =K_{m d, 0} \times \sigma_{V, 0}+\sum_{i=1}^{N}\left|S_{0}(3, i)\right| \times \max _{\text {_bias }}
\end{aligned}
$$

For checking consistency of the solution using a subset of satellites, omitting the $n^{\text {th }}$ satellite, $V P L_{n}$ reads:

$$
V P L_{n}=D_{n}+K_{m d, n} \times \sigma_{V, n}+\sum_{i=1}^{N}\left|S_{n}(3, i)\right| \times \text { max_bias }
$$

The detection threshold for the $n^{\text {th }}$ test statistic $\left(D_{\mathrm{n}}\right)$ is computed as:

$$
D_{n}=K_{f f d, n} \times \sigma_{d V, n}+\sum_{i=1}^{N}\left|\Delta S_{n}(3, i)\right| \times n o m_{-} \text {bias }
$$

$K_{m d, 0}, K_{m d, n}, K_{f f d, n}$ are scalar factors that are used to satisfy the miss-detection and the false alert probabilities and are computed from the inverse of the complement of the one-sided standard normal cumulative distribution function. $S_{0}$ and $S_{n}$ denote the projector as shown in Eq. (2) for the full-set of satellites and the subset where the $n^{\text {th }}$ satellite is excluded, and $\Delta S_{\mathrm{n}}=S_{\mathrm{n}}-S_{0}$. The nominal and maximum biases (nom_bias and max_bias) are representative of the observation quality and conditions, which are typically taken $0.1 \mathrm{~m}$ to $0.2 \mathrm{~m}$, and $0.5 \mathrm{~m}$ to 0.7 $\mathrm{m}$ respectively. $\sigma_{v, 0}, \sigma_{v, n}$ and $\sigma_{d v, n}$ are determined from the covariance matrices of the unknowns for the cases of 
full-set solution, sub-set solution, and the difference between the two.

Under operational conditions one would like to examine the observations for the presence of outliers before checking integrity of the solution. Among the widely used methods for this purpose is the Weighted Sum of the Squared Errors (WSSE) (Walter and Enge, 1995). If the test fails, identification of possible observations that may contain the faults should be performed to isolate them. Identification of faulty observations (i.e. satellites, since each satellite contributes with one ionosphere-free code observation in the solution is discussed in ElMowafy and Arora, 2013 and El-Mowafy, 2014.

When ARAIM availability requirement is met, real-time integrity can be checked using outlier-free observations by ensuring that the vertical position error is within the vertical protection level; i.e. $V P E$ is less than $V P L$. If the correct vertical position is known, for instance at reference stations, $V P E$ is computed as the difference between the estimated and known vertical position and such information can be sent to aircraft using, for instance, a Ground Based Augmentation System (GBAS). If an accurate position is not known (e.g. during flying and no GBAS), an estimate of VPE can be computed from an estimate of the observation errors, which can be computed from observation residuals (see El-Mowafy and Arora, 2013).

\section{Using GPS and BeiDou in ARAIM}

\subsection{Combining the observations of the two constellations}

For integrity checking using multiple constellations, Lee et al. (2005) and Ene et al. (2007) treated the case of GPS and Galileo by having a separate solution from each system. Choi et al. (2012) utilise a similar approach when performing ARAIM using measurements from GPS and GLONASS. In this study, observations from the two constellations (GPS and BeiDou) are used in one solution. The following points are considered in this approach:

- Measurements for the multi-constellation model refer to a unified space coordinate system by transformation of BeiDou satellite coordinates to the GPS coordinate frame.

- The receiver clock error for GPS and BeiDou are estimated for each system separately. Wu et al., discussed the case of working with time offsets between two constellations in ARAIM. Both methods should lead to the same results. The combined observation model using $u$ number of GPS satellites and $v$ number of Beidou satellites reads:

$$
\left[\begin{array}{c}
y_{u \times 1} \\
y_{v \times 1}
\end{array}\right]=\left[\begin{array}{c|cc}
G_{u \times 3} & C_{u \times 1} & 0_{u \times 1} \\
G_{v \times 3} & 0_{v \times 1} & C_{v \times 1}
\end{array}\right]\left[\begin{array}{c}
x_{3 \times 1} \\
d t_{G P S} \\
d t_{B e i D o u}
\end{array}\right]+\left[\begin{array}{c}
\varepsilon_{u \times 1} \\
\varepsilon_{v \times 1}
\end{array}\right]
$$

where the subscripts refer to the size of the matrices or vectors, $G_{u \times 3}$ and $G_{v \times 3}$ refer to the first three column elements of the $G$ matrix corresponding to the satellite sets $u$ and $v, C$ is a unit column vector, $d t_{G P S}$ and $d t_{\text {BeiDou }}$ denote the receiver clock errors for GPS and BeiDou systems, respectively.

- Since BeiDou and GPS currently broadcast on different frequencies, there is no need for estimation of the inter-system biases.

- The broadcast total group delay of BeiDou TGD1(B1/B3) and TGD2 (B2/B3) are used in place of the differential code biases (see Montenbruck et al. 2013).

- Ionosphere-free linear combination can be performed for GPS satellites broadcasting L1 and L5 signals (or using L1 and L2 for satellites with no L5 signals for testing purposes). For BeiDou, ionosphere-free linear combination is formed using B1 and B2 frequencies, and using its In-phase "I" component, which is likely to be intended for BeiDou open service whereas the "Q" component is planned for authorized users (Grelier et al. 2007).

\subsection{Fault probabilities for GPS and BeiDou}

The chosen probability of Hazardously Misleading Information ( $\operatorname{Pr}\{\mathrm{HMI}\})$, the a-priori risk probability $\left(\mathrm{P}_{\text {aappriori }}\right)$, the false alarm probability $\left(\mathrm{P}_{\mathrm{fa}}\right)$, and missed detection probability $\left(\mathrm{P}_{\mathrm{md}}\right)$ for different cases of faults (single or multiple faults for one constellation or more) are critical parameters for computation of the detection and identification test statistics, the protection levels and their thresholds. They accordingly affect the FDE and integrity performance in general. The corresponding values for GPS are well established and are already in use by existing RAIM receivers. The United States has made very specific performance commitments for GPS to provide assurance that the RAIM assumptions will be met, from which the $\mathrm{P}\{\mathrm{HMI}\}$ and fault-probabilities were assumed for GPS. To date, no other constellation provider has delivered similar assurances. For BeiDou, similar basis for assumptions on fault-probabilities are not yet available and will need further investigation and international coordination over a long period of time. Recently China Satellite Navigation Office (2013) has released the first BeiDou Navigation Satellite System Open Service Performance Standard (BDS-OS-PS-1.0) to provide information on how the system is going to be operated in the future. This would serve as the first base for determining the appropriate degree of trust that should be placed in BeiDou. 
Standard Positioning Service - Performance Standard (SPS PS) of GPS has provided assurances that there would not be more than three major service failures per year for the GPS constellation as a whole. This confirms with the historical frequency of GPS signal faults (GEAS, 2010), where GPS historical record shows the ability to remove faulted signals in less than one hour (Wlater et al., 2013). Hence, for GPS, the $\mathrm{P}_{\mathrm{a} \text {-priori }}$ value is assumed taking into consideration a frequency that is equivalent to a probability onset of about $1 \times 10^{-5}$ per satellite per hour $(\approx 3 /[31$ sat $\times 365$ day $\times 24 \mathrm{hr}])$, approximated to per approach.

The false alert probability for vertical guidance is taken equal $4 \times 10^{-6}$ per 15 -second interval, which was derived from the ICAO continuity risk requirement (ICAO, 2009). The allowable false alert probability per sample is also taken the same as the probability per 15-second interval at $4 \times 10^{-6}$ (Blanch et al., 2010). This value is assumed for both GPS and BeiDou.

The definition of a fault in BeiDou is currently open to interpretation; therefore, BeiDou fault rates used would be more indicative than precise. In this study, $\mathrm{P}_{\text {a-priori }}$ of BeiDou is assumed $1 \times 10^{-4}$ per satellite per hour (approximated to per approach) as a conservative measure, which will be further assessed with time by monitoring performance of the system. A major service failure is assumed in the event that the instantaneous UERE > 4.42 URA (broadcast) for GPS block II satellites or UERE > $5.73 \times$ URA for GPS block III satellites when the satellite is set "healthy" without a timely alert is being issued (Shively, 2009). Since BeiDou has a modern design with three operational frequencies, which allows for forming a ionosphere-free linear combination of its observations, a major service failure is assumed when UERE > 5.73×URA. This provided a tight bound on the error as the URA is most often set in the navigation message to $2 \mathrm{~m}, 2.4 \mathrm{~m}$ and 3.6 $\mathrm{m}$ for GPS, where currently the most noted value is 2.4 $\mathrm{m}$. For BeiDou, the URE is set between $2 \mathrm{~m}$ and $2.4 \mathrm{~m}$ in the navigation message, where it is not known if this value represents actual or predictive status. URA is not given and it was assumed $2.4 \mathrm{~m}$ in this study.

Safety is assured if the sum of the product of the missed detection and prior probabilities is below the $\operatorname{Pr}\{\mathrm{MI}\}$ requirement. Therefore, the modes that has two or more independent single GPS satellite faults, or that couple a single GPS fault with a single or multiple BeiDou faults are considered to have very small likelihood and may avoid needing availability evaluation provided there are not too many satellites. They are however included in the integrity calculation. Therefore, in the assessment of availability, we evaluate the no fault mode (all satellites included in position estimate) with a conservatively assigned prior probability of one. We also evaluate each individual GPS and BeiDou satellite removed subset. Finally, we evaluate one subset in which all BeiDou satellites are removed from the estimate with the conservatively assigned prior probability, thus, creating a subset that is not at all affected by any BeiDou fault mode. This test evaluates not only the constellation wide fault mode, but any fault mode that affects any number of BeiDou satellites. The VPL value used in checking availability is selected as the largest amongst all these cases.

\section{Testing and data analysis}

\subsection{Test description}

To evaluate ARAIM availability, some studies use simulated data over a global grid, however, integrity is rather intended as real time decision criterion for using or not using the system. Therefore, validation of ARAIM integrity and availability components using real data is essential. Thus, in this study, testing was performed using real measurements from eight globally distributed Continuously Operating Reference Stations (CORS) in Australia, China, Netherlands, eastern Canada and Peru. Sites in Australia include stations CUT0 at Curtin University in Western Australia, and stations MNGO, SWNH and WORI of Vicmap Position - GPSnet in Victoria (East Australia). Stations JFNG was used in China, station DELF1 at Delft University of Technology, the Netherlands, station UNB3 at the University of New Brunswick in Canada and station AREG in Peru were also used. Figure 2 shows the approximate locations of the test sites. The data at each station span two consecutive days (30/5/2013 and 1/6/2013) with a sampling rate of 30 seconds and a mask angle of 5 degrees. The data were obtained from the multi-GNSS experiment (MGEX) and GPSnet online archives. Stations in Australia and China were capable of tracking all BeiDou satellites. However, as BeiDou constellation has currently a regional coverage over Asia-Pacific area including oceania, stations DELF1 and UNB3 were only capable of tracking some of the GEO and IGSO satellites. Station UNB3 tracked satellite C07 (IGSO), whereas station DELF1 tracked satellites C02, C05 (GEO), C06, C07, C09 and C10, which are IGSO. Station AREG cannot track any GEO or IGSO satellites but tracked, as well as stations DLF1 and UNB3, all four MEO satellites available during the time of testing.

In the test, the complete integrity monitoring process was executed, including ARAIM availability, FDE, and integrity testing. A comparison between ARAIM performance with the use of GPS and GPS+BeiDou was performed. Because testing was carried out at sites with known positions, in computation of the VPE the GNSS determined positions were compared with the known locations of the antennas. The VPL was computed based 
on the assumed accuracy parameters, fault probabilities and satellite geometry at each site.

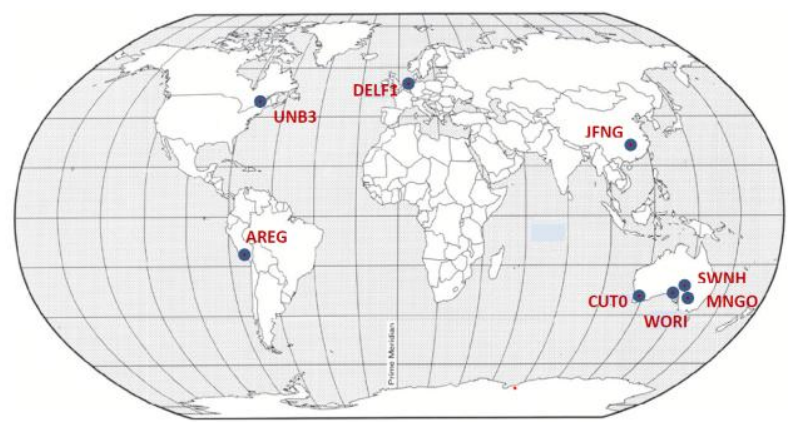

Figure 2: Approximate locations of the test sites

\subsection{Test results}

Figures 3 and 4 show, as an example, integrity monitoring parameters VPE-VPL-VAL at station CUT0 determined from data collected on 30/5/2013. They were computed first using GPS data only (Figure 3) and then using combined GPS+BeiDou observations (Figure 4). Figure 5 shows the number of GPS and BeiDou satellites observed during the same period. This number affects availability of ARAIM and its performance. As the figures show, when the number of observed satellites is low; $V P L$ is generally high and may exceed $V A L$, indicating no availability of ARAIM. As Figure 3 illustrates, ARAIM was unavailable at several epochs using only GPS data. However, when GPS observations are augmented with BeiDou measurements, $V P L$ is reduced and hence ARAIM was available at all the time due to the increased number and better geometry of satellites used in computation of VPL.

Figures 6 and 7 show another example on the impact of the inclusion of BeiDou satellites into the estimation of integrity parameters at station MNGO in Victoria on $30 / 5 / 2013$, which results in a substantial improvement to availability performance. As can be seen from Figure 7, the $V P L$ is significantly reduced, leading to significantly fewer incidents of unavailability of ARAIM with the addition of the BeiDou constellation, despite the decreased confidence placed in its performance compared with GPS. For other stations, the availability results vary due to the varying number of satellite observations, their geometry and the specific conditions at each site. Overall, ARAIM availability has increased up to $8 \%$ when adding BeiDou to GPS satellites.

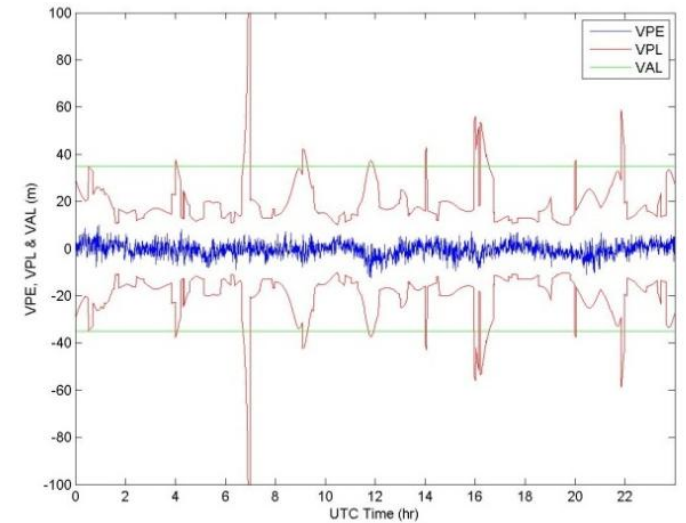

Figure 3: Intigrity Monitoring using GPS at CUT0

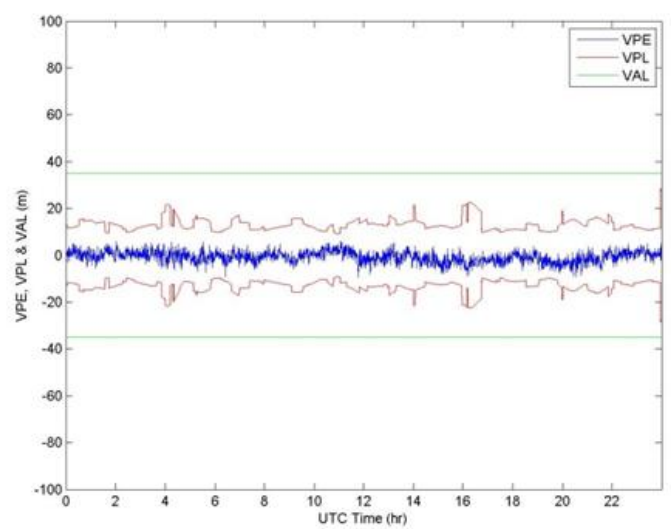

Figure 4: ARAIM using GPS+BeiDou at CUT0

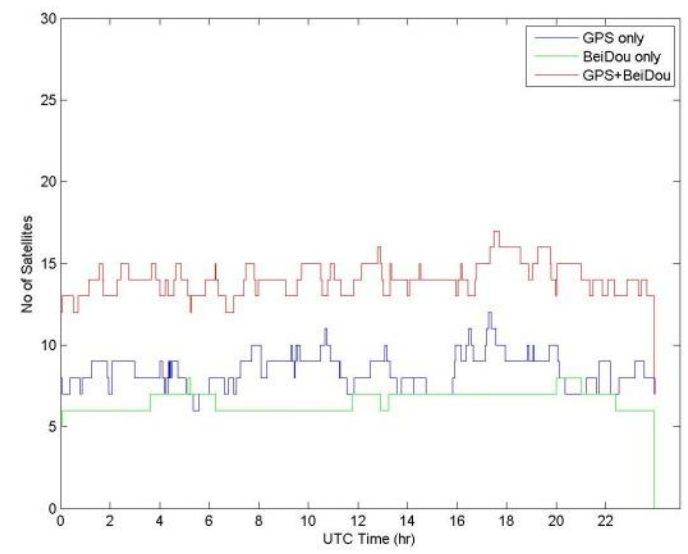

Figure 5: Number of Satellites at CUT0 on 30/5/2013 


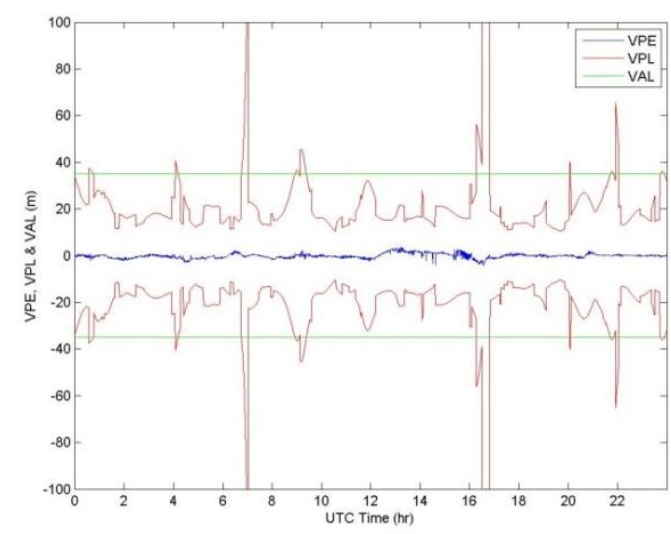

Figure 6: Intigrity Monitoring using GPS only at MNGO

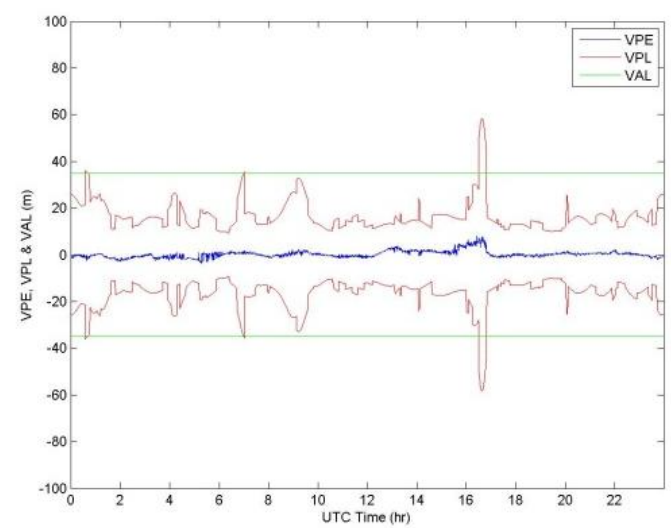

Figure 7: IntgrityMonitoring using GPS+BeiDou at MNGO

Interestingly, station AREG in Peru which is unable to track GEO or IGSO satellites did not suffer from unavailability of ARAIM using GPS alone as depicted in Figure 8, and thus adding BeiDou does not significantly improve availability of ARAIM in general. This can be attributed to its closeness to the Equator and the better GPS satellite geometry at this location.

Table 1: Comparison between mean and standard deviation (m) of VPE using GPS and GPS+BeiDou

\begin{tabular}{|l|c|c|c|c|}
\hline \multirow{2}{*}{ Station } & \multicolumn{2}{|c|}{ GPS } & \multicolumn{2}{c|}{ GPS+BeiDou } \\
\cline { 2 - 5 } & $\mu_{V P E}$ & $\sigma_{V P E}$ & $\mu_{V P E}$ & $\sigma_{V P E}$ \\
\hline CUTO & 0.101 & 0.726 & 0.060 & 0.659 \\
\hline MNGO & -0.054 & 0.964 & 0.187 & 1.365 \\
\hline SWNH & 0.094 & 1.448 & 0.050 & 1.321 \\
\hline WORI & -0.002 & 1.218 & 0.005 & 1.115 \\
\hline DLF1 & 0.001 & 1.019 & 0.001 & 0.897 \\
\hline UNB3 & -0.118 & 0.843 & -0.144 & 0.951 \\
\hline JFNG & -0.110 & 1.147 & -0.003 & 1.050 \\
\hline AREG & 0.019 & 1.0966 & 0.017 & 1.083 \\
\hline
\end{tabular}

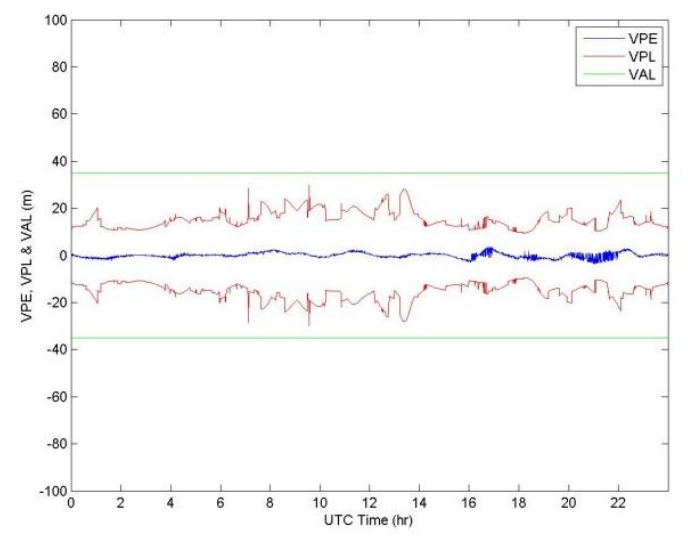

Figure 8: Intigrity Monitoring using GPS only at AREG

For integrity testing, the focus is on keeping the $V P E$ bounded by the VPL. Figure 9 and 10 show, as an example, histograms of the computed $V P E$, its mean $\left(\mu_{V P E}\right)$ and standard deviation $\left(\sigma_{V P E}\right)$ using firstly GPS data only and secondly using GPS+BeiDou data collected at CUT0, respectively. As the figures display, most VPE values were bounded within $\pm 2 \mathrm{~m}$, and as expected, follow a normal distribution with a mean close to zero. The standard deviation of the VPE using GPS+BeiDou mode was less than that for GPS only mode in this case indicating better positioning results. However, the improvement in positioning accuracy (i.e. $V P E$ values) due to adding BeiDou measurements varied across the tested sites as given in Table 1 according to number and quality of the received BeiDou observations. Some BeiDou satellites have also experienced frequent clock jumps. Even in the cases when the use of BeiDou observations with GPS gave VPE slightly higher than using GPS alone, the VPE were well bounded within the $V P L$ as shown in Figure 7 for station MNGO. When studying the VPE/VPL ratio as shown in Figures 11 and 12 for station CUT0, it was in general within \pm 0.2 . Note here that $V P E / V P L$ ratio was only computed when ARAIM was available, therefore, due to the fact that this availability was higher in case of GPS+BeiDou than when using only GPS, the sample size shown in the figures for GPS+BeiDou was more than that for GPS only.

To conclude, the paper has shown some preliminary evaluation of integrity monitoring using GPS and GPS+BeiDou. There is still much work left to be done in our future work. We want to verify our implementation with many more cases, including simulated faults, to ensure that the algorithm is behaving as expected. In addition, we need to test at more sites. An important point is that constellations like BeiDou needs comprehensive error modelling and long-term monitoring of its performance to allocate more concrete values to fault probabilities. 


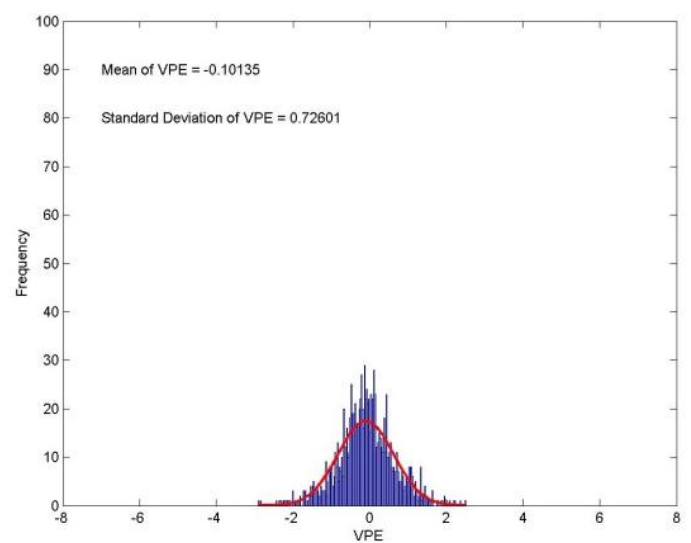

Figure 9: VPE (m) histogram using GPS alone

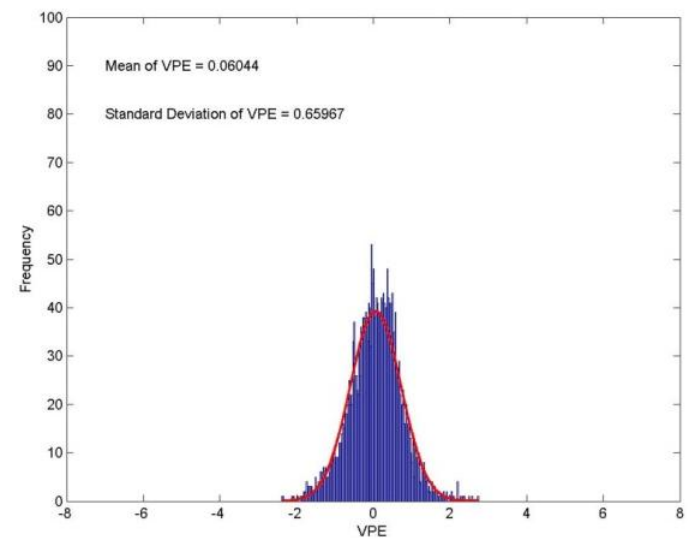

Figure 10: $V P E(\mathrm{~m})$ histogram using GPS+BeiDou

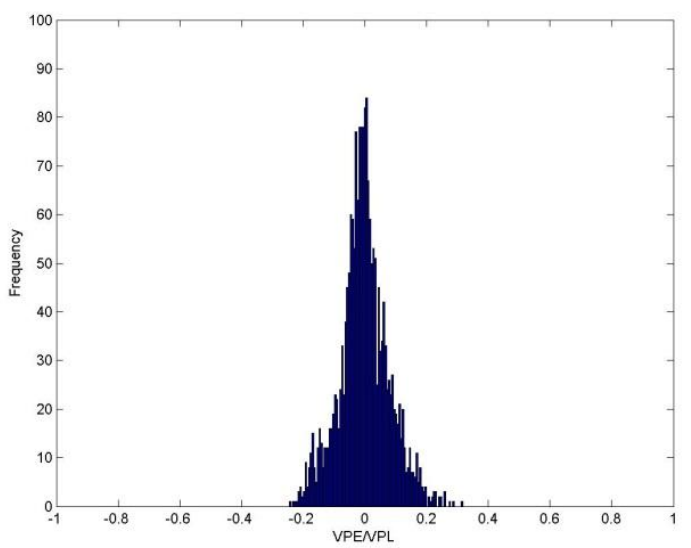

Figure 11: VPE/VPL ratio using GPS alone

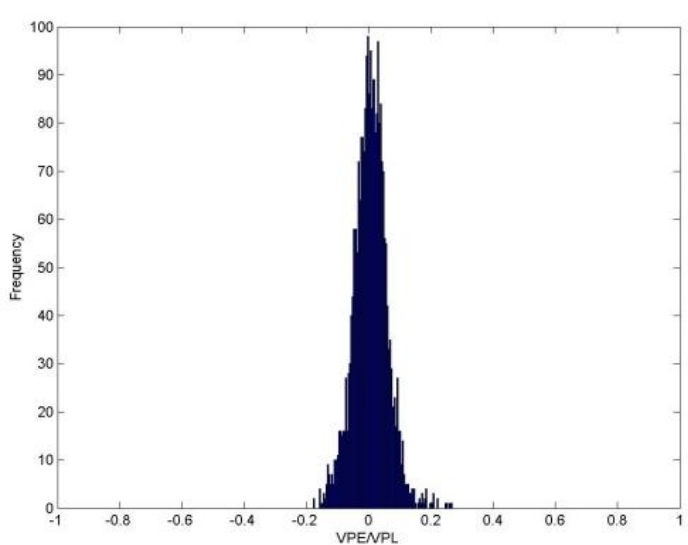

Figure 12: VPE/VPL ratio using GPS+BeiDou

\section{Conclusions}

An ARAIM method with focus on vertical guidance is presented, which included three steps: checking availability of ARAIM, fault detection and exclusion, and integrity checking. The case of using observations from two GNSS constellations, namely GPS and BeiDou is investigated, where their observations are combined in one ARAIM solution. Parameters that are utilised for the integration of the two systems' observations were discussed. In addition, allocation of the probability of Hazardously Misleading Information for most likely types of faults of a combined GPS and BeiDou measurements model are presented.

Comparison between the use of GPS and GPS+BeiDou ARAIM was performed using real data over two days at eight CORS sites with global distribution. Results showed that using GPS with BeiDou has improved availably of ARAIM compared with GPS alone. The improvement in positioning accuracy, assessed in terms of the VPE values, due to adding BeiDou measurements varied across the tested sites. Even in the cases when the use of BeiDou observations with GPS gave $V P E$ slightly higher than GPS alone, with $V P E$ computed as the difference between the estimated and known vertical position, most $V P E$ values were constrained within $\pm 2 \mathrm{~m}$ and were well bounded within the $V P$, indicating acceptable integrity.

\section{Acknowledgments}

Balwinder Arora is acknowledged for this participation in coding one module of the software used in this study. MGEX and Vicmap Position - GPSnet (Victoria) are acknowledged for providing the GPS and BeiDou data used. 


\section{References}

Blanch J., A. Ene, T. Walter, and P. Enge (2007), An Optimized Multiple Hypothesis RAIM Algorithm for Vertical Guidance, Proceedings of the ION GNSS 2007, Fort Worth, TX, Sept. 25-28, pp. 2924-2933.

Blanch J., T. Walter, and P. Enge (2010), RAIM with Optimal Integrity and Continuity Allocations under Multiple Failures, IEEE Transactions on Aerospace and Electronic Systems, Vol. 46, No. 3, pp. 12351247.

Brown R.G. (1992), A baseline GPS RAIM scheme and a note on the equivalence of three RAIM methods, Navigation, Vol. 39, No. 3, pp. 301-316.

China Satellite Navigation Office (2013), BeiDou Navigation Satellite System Open Service Performance Standard (BDS-OS-PS-1.0).

Choi M., J. Blanch, T. Walter, and P. Enge (2011a), Advanced ARAIM Demonstration using Four Months of Ground Data, Proceedings of the International Technical Meeting of the Institute of Navigation, San Diego, CA, 24-26 Jan. 2011, pp. 279-284.

Choi M., J. Blanch, T. Walter, and P. Enge (2011b), Demonstrations of Multi-Constellation Advanced RAIM for Vertical Guidance using GPS and GLONASS Signals, Proceedings of ION GNSS 2011, Portland, OR, 2011, pp. 3227-3234.

Choi M., Blanch J., Walter T., Akos D., and P. Enge (2012), Evaluation of Multi-Constellation Advanced RAIM for Vertical Guidance using GPS and GLONASS Signals with Multiple Faults, Proceedings $25^{\text {th }}$ Interntional Technical Meeting, ION, Nashville TN, Sept. 17-21, 2012, p. 884-892.

El.Mowafy A. (2013), Real-Time Validation of BeiDou Observations in a Stand-alone Mode, Proceedings of ION Pacific PNT conference, Honolulu, Hawaii, April 22-25, 2013, pp. 106-114.

El.Mowafy A., and B. Arora (2013), The Current ARAIM Availability According to LPV-200 Using GPS and BeiDou in Western Australia, Proceedings of International Global Navigation Satellite Systems Society (IGNSS) Symposium 2013, Gold Coast, 16-18 July, 2013, pp. 1-16.
El-Mowafy, A. (2014), GNSS Multi-frequency Receiver Single-Satellite Measurement Validation Method, GPS Solutions, Published online DOI 10.1007/s10291-013-0352-6.

El-Mowafy, A. (2005). Decimeter Level Mapping Using Differential Phase Measurements of GPS Handheld Receivers, Survey Review, Vol. 38, No. 295, 47-57.

El-Mowafy, A., (2011). Analysis of the Web-Based PostProcessing GNSS Services for Static and Kinematic Positioning in Surveying Applications of Short Data Spans, Survey Review, 43-323, pp. 535-549.

Ene A., J. Blanch, and P.J. David (2007), Fault detection and elimination for Galileo-GPS vertical guidance, Proceedings of the 2007 National Technical Meeting of the Institute of Navigation, San Diego, CA, 22-24 Jan., 2007 pp. 1244-1254.

Geng Y., Li R., Xu H., and X. Zhan (2010), Performance enhancement in BeiDou system Integrity and Accuracy. BeiDou Integrity and Accuracy, Proceedings ICG WG-B Meeting, Oct. 2010, Torino, Italy, pp. 1-26.

GNSS Evolutionary Architecture Study Phase II Report (GEAS) (2010), http://www.faa.gov/ about/office_org/headquarters_offices/ato/service_un its/techops/navservices/gnss/library/documents.

GNSS Evolutionary Architecture Study Phase I Panel Report (GEAS) (2008), http://www.faa.gov/about/office_org/headquarters_of fices/ato/service_units/techops/navservices/gnss/libra ry/documents.

Grelier T, Dantepal J., DeLatour A., Ghion A., and L. Ries (2007), Initial Observations and Analysis of Compass MEO Satellite Signals, Inside GNSS, Vol. 5, No. 6, pp. 39-43.

Guo J., M. Lu, X. Cui, and Z. Feng (2011), A New RAIM Algorithm for Triple-frequency GNSS Receivers, Proceedings ION International Technical Meeting, San Diego, CA, January 24-26, pp. 271278.

Hofmann-Wellenhof B., H. Lichtenegger, E. Wasle (2008), GNSS - Global Navigation Satellite Systems : GPS, GLONASS, Galileo, and more. SpringerVerlag, Vienna. 
Hwang P., and R.G. Brown (2008), From RAIM to NIORAIM - A New Integrity Approach to Integrated Multi-GNSS Systems, Inside GNSS, Vol. 3, No. 3, pp. 24-33.

ICAO, Annex 10 (2009), GNSS standards and recommended practices (SARPs), in Section 3.7, Appendix B, and Attachment D, Aeronautical Telecommunications, Vol. 1 (Radio Navigation Aids), Amendment 84, November 2009.

Kneiß1 F., C. Stöber, and B. Eissfeller (2009), Assessment of Combined Integrity Algorithms. Proceedings of the 22nd International Technical Meeting of The Satellite Division of the Institute of Navigation (ION GNSS 2009), Savannah, GA, September 2009, pp. 2804-2817.

Lee Y., R. Braff, J.P. Fernow, D. Hashemi, M.P. McLaughlin, and D. O'Laughlin (2005), GPS and Galileo with RAIM or WAAS for Vertically Guided Approaches, Proceedings of the $18^{\text {th }}$ International Technical Meeting of the Satellite Division of The Institute of Navigation (ION GNSS2005), 13-16 Sept. 2005, Long Beach, CA, pp. 1801-1825.

Lee C., and M. McLaughlin (2007), Feasibility Analysis of RAIM to Provide LPV-200 Approaches with Future GPS, Proceedings of the ION GNSS 2007, Fort Worth, TX, 2007, Sept. 25-28, pp. 2898-2910.

Lee Y. (2012), New Advanced RAIM with Improved Availability for Detecting Constellation-wide Faults Using Two Independent Constellations, Navigation, Vol. 60, No. 1, pp. 71-83.

Lijun P., J. Kai, D. Xiaojun, Z. Yan, and Y. Wenqiang (2012), Receiver Autonomous Integrity Monitoring Parameter Design and Analysis for MultiConstellation Navigation, Proceedings China Satellite Navigation Conference (CSNC) 2012 (2), pp. 15-28.

Montenbruck O., P. Steigenberger, R. Khachikyan, G. Weber, R.B. Langley, L. Mervart, and U. Hugentobler (2013), IGS-MGEX: Preparing the Ground for Multi-Constellation GNSS Science, $4^{\text {th }}$ International Colloquium on Scientific and Fundamental Aspects of the Galileo System, Prague, 4-6 Dec 2013, pp. 1-8.
Shively C. (2009), Analysis of Specified and Hypothetical GPS IIIC Integrity for LPV 200 Operations, Publications of The MITRE Corporation, pp.1-12, http://www.mitre.org/ sites/default/ files/pdf/09_5396.pdf.

US Federal Radionavigation Plan (US FRP) (2008), Report No. DOT-VNTSC-RITA-08-02/DoD-4650.5.

Walter T., and P. Enge (1995), Weighted RAIM for Precision Approach, Proceedings of the ION-GPS 1995, Palm Springs, CA, September 1995, pp. 499509.

Walter T., J. Blanch, M.J. Choi, T. Reid, and P. Enge (2013), Incorporating GLONASS into Aviation RAIM Receivers, Proceedings of the 2013 International Technical Meeting of the Institute of Navigation, San Diego, CA, 27-29 January, 2013, pp. 239-249.

Wu Y., Wang J., and Jiang Y. (2013), Advanced receiver autonomous integrity monitoring (ARAIM) schemes with GNSS time offsets, Advances in Space Research, vol. 52 , no. 1 , pp. $52-61$.

\section{Biography}

Dr. Ahmed El-Mowafy is a Senior Lecturer, Department of Spatial Sciences, Curtin University, Australia. He has obtained his Ph.D. from the University of Calgary, Canada, in 1995. He has extensive publications in precise positioning and navigation using GNSS, quality control and integrity monitoring, estimation theory, PPP and attitude determination. He is a member of the Editorial Advisory Board of the Journal of Navigation. a.el-mowafy@curtin.edu.au 